\title{
Fundamental characteristics and high-speed applications of carrier-depletion silicon Mach-Zehnder modulators
}

\author{
Kensuke Ogawa $^{1 \text { a) }}$, Hiroki Ishihara ${ }^{1}$, Kazukiro Goi ${ }^{1}$, \\ Yasuhiro Mashiko ${ }^{1}$, Soon Thor $\mathbf{L i m}^{2}$, Min Jie Sun ${ }^{3}$, \\ Sean Seah ${ }^{3}$, Ching Eng Png ${ }^{2}$, Tsung-Yang Liow ${ }^{4}$, \\ Xiaoguang $\mathbf{T u}^{4}$, Guo-Qiang $\mathbf{L o}^{4}$, and Dim-Lee Kwong ${ }^{4}$ \\ ${ }^{1}$ Optics and Electronics Laboratory, Fujikura Ltd., \\ 1440 Mutsuzaki, Sakura, Chiba 285-855, Japan \\ ${ }^{2}$ Institute of High-Performance Computing, A*STAR, \\ 1 Fusionopolis Way, Singapore 138632, Singapore \\ ${ }^{3}$ Optic2Connect Pte Ltd., \\ 71 Ayer Rajah Crescent, \#03-07, Singapore 139951, Singapore \\ ${ }^{4}$ Institute of Microelectronics, $A * S T A R$, \\ 11 Science Park Road, Singapore Science Park II, Singapore 117685, Singapore \\ a) kensuke.ogawa@jp.fujikura.com
}

\begin{abstract}
Carrier-depletion Si Mach-Zehnder modulators incorporating lateral PN-junction phase shifters are reviewed in the lights of fundamental characteristics and applications to high-speed optical fiber transmission. Experimental reverse-bias characteristics are supported by numerical analysis with good agreement, implying that the Si modulators are fabricated precisely as designed. Numerical high-speed response proves that the phase shifter operates beyond 100-Gbaud symbol rate. Traveling-wave electrodes are characterized in S-parameter measurements to reveal $L R C$ resonance as a limiting factor in high-speed modulation. High-speed optical-fiber transmission in on-off and phase-shift keying formats is demonstrated using the Si Mach-Zehnder modulators assembled in ceramic-based metal packages. Keywords: optical modulator, photonic integrated circuit, Mach-Zehnder interferometer, on-off keying, phase-shift keying, silicon photonics
\end{abstract}

Classification: Optoelectronics, Lasers and quantum electronics, Ultrafast optics, Silicon photonics, Planar lightwave circuits

\section{References}

[1] F. Koyama and K. Iga: J. Lightwave Technol. 6 (1988) 87. DOI:10.1109/50.3969

[2] A. H. Gnauck and P. J. Winzer: J. Lightwave Technol. 23 (2005) 115. DOI:10. 1109/JLT.2004.840357

[3] P. J. Winzer and R.-J. Essiambre: Proc. IEEE 94 (2006) 952. DOI:10.1109/ JPROC. 2006.873438

[4] K. Kikuchi: ECOC Digest (2008) Th.2.A.1. DOI:10.1109/ECOC.2008.4729551 
[5] G. T. Reed, G. Mashanovich, F. Y. Gardes and D. J. Thomson: Nat. Photonics 4 (2010) 518, and references therein. DOI:10.1038/nphoton.2010.179

[6] T.-Y. Liow, K.-W. Ang, Q. Fang, J.-F. Song, Y.-Z. Xiong, M.-B. Yu, G.-Q. Lo and D.-L. Kwong: IEEE J. Sel. Top. Quantum Electron. 16 (2010) 307. DOI:10.1109/ JSTQE.2009.2028657

[7] D. Guckenberger, S. Abdalla, C. Bradbury, J. Clymore, P. De Dobbelaere, D. Foltz, S. Gloeckner, M. Harrison, S. Jackson, D. Kucharski, Y. Liang, C. Lo, M. Mack, G. Masini, A. Mekis, A. Narasimha, M. Peterson, T. Pinguet, J. Redman, S. Sahni, B. Welch, K. Yokoyama and S. Yu: ECOC Digest (2010) Tu.4.C.2. DOI:10. 1109/ECOC.2008.4729551

[8] O. Mikami and H. Nakagome: Electron. Lett. 20 (1984) 228. DOI:10.1049/ el: 19840153

[9] L. Friedman, R. A. Soref and J. P. Lorenzo: J. Appl. Phys. 63 (1988) 1831. DOI: $10.1063 / 1.339878$

[10] R. A. Soref and B. R. Bennett: IEEE J. Quantum Electron. 23 (1987) 123. DOI:10. 1109/JQE.1987.1073206

[11] F. Y. Gardes, G. T. Reed, N. G. Emerson and C. E. Png: Opt. Express 13 (2005) 8845. DOI:10.1364/OPEX.13.008845

[12] B. Analui, D. Guckenberger, D. Kucharski and A. Narasimha: IEEE J. Solid-State Circuits 41 (2006) 2945. DOI:10.1109/JSSC.2006.884388

[13] K. Goi, K. Oda, H. Kusaka, Y. Terada, K. Ogawa, T.-Y. Liow, X. Tu, G.-Q. Lo and D.-L. Kwong: Opt. Express 20 (2012) B350. DOI:10.1364/OE.20.00B350

[14] A. Liu, L. Liao, D. Rubin, H. Nguyen, B. Ciftcioglu, Y. Chetrit, N. Izhaky and M. Paniccia: Opt. Express 15 (2007) 660. DOI:10.1364/OE.15.000660

[15] B. Milivojevic, C. Raabe, A. Shastri, M. Webster, P. Metz, S. Sunder, B. Chattin, S. Wiese, B. Dama and K. Shastri: OFC/NFOEC Digest (2013) OTh1D.1.

[16] K. Ogawa, K. Goi, Y. T. Tan, T.-Y. Liow, X. Tu, Q. Fang, G.-Q. Lo and D.-L. Kwong: Opt. Express 19 (2011) B26. DOI:10.1364/OE.19.000B26

[17] H. Ishihara, K. Oda, T. Ori, K. Goi, K. Ogawa, T.-Y. Liow, X. Tu, G.-Q. Lo and D.-L. Kwong: ICEP Proc. (2014) FE2-1. DOI:10.1109/ICEP.2014.6826765

[18] H. Kusaka, A. Oka, K. Goi, K. Ogawa, T.-Y. Liow, X. Tu, G.-Q. Lo and D.-L. Kwong: OECC Digest (2013) MM1-4.

[19] C. E. Png, V. Dixit, S. T. Lim and E.-P. Li: Proc. SPIE 8629 (2013) 86290S. DOI: $10.1117 / 12.2002696$

[20] C. E. Png, S. P. Chan, S. T. Lim and G. T. Reed: J. Lightwave Technol. 22 (2004) 1573. DOI:10.1109/JLT.2004.827655

[21] A. K. Jonscher and M. H. Boyle: Int. Symp. GaAs Digest (1966) 12A.

[22] K. Seeger: Semiconductor Physics (Springer, New York, 1973) 240.

[23] D. J. Thomson, F. Y. Gardes, Y. Hu, G. Mashanovich, M. Fournier, P. Grosse, J.-M. Fedeli and G. T. Reed: Opt. Express 19 (2011) 11507. DOI:10.1364/OE.19. 011507

[24] P. Dong, L. Chen and Y.-K. Chen: Opt. Express 20 (2012) 6163. DOI:10.1364/OE. 20.006163

[25] K. Ogawa, K. Goi, H. Kusaka, Y. Terada, T.-Y. Liow, X. Tu, G.-Q. Lo, D.-L. Kwong, V. Dixit, S. T. Lim and C. E. Png: Proc. SPIE 8629 (2013) 86290U. DOI: $10.1117 / 12.2002311$

[26] P. Dong, C. Xie, L. Chen, L. L. Buhl and Y.-K. Chen: Opt. Express 20 (2012) B624. DOI:10.1364/OE.20.00B624

[27] K. Goi, H. Kusaka, A. Oka, K. Ogawa, T.-Y. Liow, X. Tu, G.-Q. Lo and D.-L. Kwong: OFC Digest (2014) W1I.2.

[28] H. Yu and W. Bogaerts: J. Lightwave Technol. 30 (2012) 1602. DOI:10.1109/JLT. 2012.2188779 
[30] T. Mizuochi, K. Ishida, T. Kobayashi, J. Abe, K. Kinjo, K. Motoshima and K. Kasahara: J. Lightwave Technol. 21 (2003) 1933. DOI:10.1109/JLT.2003.816849

[31] Q. Fang, T.-Y. Liow, J. F. Song, C. W. Tan, M. B. Yu, G. Q. Lo and D.-L. Kwong: Opt. Express 18 (2010) 7763. DOI:10.1364/OE.18.007763

[32] K. Goi, K. Oda, Y. Mashiko, K. Ogawa, T.-Y. Liow, X. Tu, G.-Q. Lo and D.-L. Kwong: ECOC Digest (2014) P.2.20. DOI:10.1109/ECOC.2014.6964199

\section{Introduction}

Mach-Zehnder (MZ) optical modulators are crucial in optical transport networks of high transmission capacity and high spectral efficiency, because they are capable of high-contrast optical modulation without deterioration due to spectral broadening and frequency chirping [1, 2, 3, 4]. Design and fabrication technologies based on silicon photonics allow monolithic integration of MZ modulators with photonic devices such as photodiodes (PDs) in small footprints [5, 6, 7]. Free-carrier plasma dispersion is the principal mechanism for high-speed refractive-index modulation in Si modulators. Free-carrier plasma dispersion was exploited initially to carrierinjection InGaAsP/InP optical switches and thereafter to carrier-injection Si optical modulators $[8,9]$. Free-carrier plasma dispersion was studied to provide experimental relationship between refractive index and carrier concentration in crystalline $\mathrm{Si}$ in a broad spectral range extending over $\mathrm{C}$ and $\mathrm{L}$ bands [10]. The empirical formula has been used to design and model a variety of Si phase shifters [5]. Carrier depletion in reverse-biased PN junction is effective to induce fast carrier displacement for high-speed modulation at symbol rates beyond 10 Gbaud [11]. Carrierdepletion Si MZ modulators incorporating lateral PN-junction phase shifters were fabricated using CMOS-compatible fabrication processes on silicon-on-insulator (SOI) wafers [12]. The lateral PN-junction carrier-depletion Si MZ modulators have an advantage in phase-shift keying (PSK) over the other types of Si MZ modulators because of weak phase nonlinearity on modulation voltage with negligible carrierinduced intensity modulation [13]. Lateral PN-junction Si modulators are also advantageous in high-yield low-cost fabrication, because its fabrication does not require deposition or epitaxy such as to form vertical $\mathrm{PN}$-junction or capacitor structures $[5,14,15]$.

This article reviews lateral PN-junction carrier-depletion monolithic Si MZ modulators. Simulation of DC voltage characteristics in terms of voltage dependent phase shifts and carrier-induced optical loss and high-speed characteristics are validated by experimental measurements with both showing good agreement. This implies the Si modulators are fabricated precisely as designed. Numerical analysis on high-speed carrier dynamics proves the phase shifter operates beyond 100Gbaud symbol rate. Traveling-wave electrodes are characterized in S-parameter measurements, which reveal $L R C$ resonance as a limiting factor in high-speed modulation. Optical-fiber transmission using the Si MZ modulators assembled in ceramic-based metal packages is demonstrated in 10-Gb/s nonreturn-to-zero (NRZ) on-off keying (OOK) and 128-Gb/s dual-polarization quadrature phase-shift keying (DP-QPSK). 


\section{Lateral PN-junction Si MZ modulator}

A traveling-wave lateral PN-junction monolithic Si MZ modulator consisting of single MZ interferometer (MZI), monitor Ge PD and RF coplanar waveguide (CPW) on the MZI is illustrated with a cross-section photograph of a rib-waveguide phase shifter and a top-view photograph of an entire modulator chip in Fig. 1. The single MZI is suitable for application to modulators operated in NRZ OOK formats. Lateral PN junction was formed in Si rib-waveguide phase shifters with length $\ell=4 \mathrm{~mm}$ in the both arms of MZI. The rib waveguide was designed with the dimensions of 500- or 600-nm rib width, 220-nm rib height and 95-nm side slab height for transverse-electric (TE) polarization. Vertical boundary of PN junction is positioned in the middle of the rib. The dimensions were determined with respect to high modulation speed and low optical loss [16].

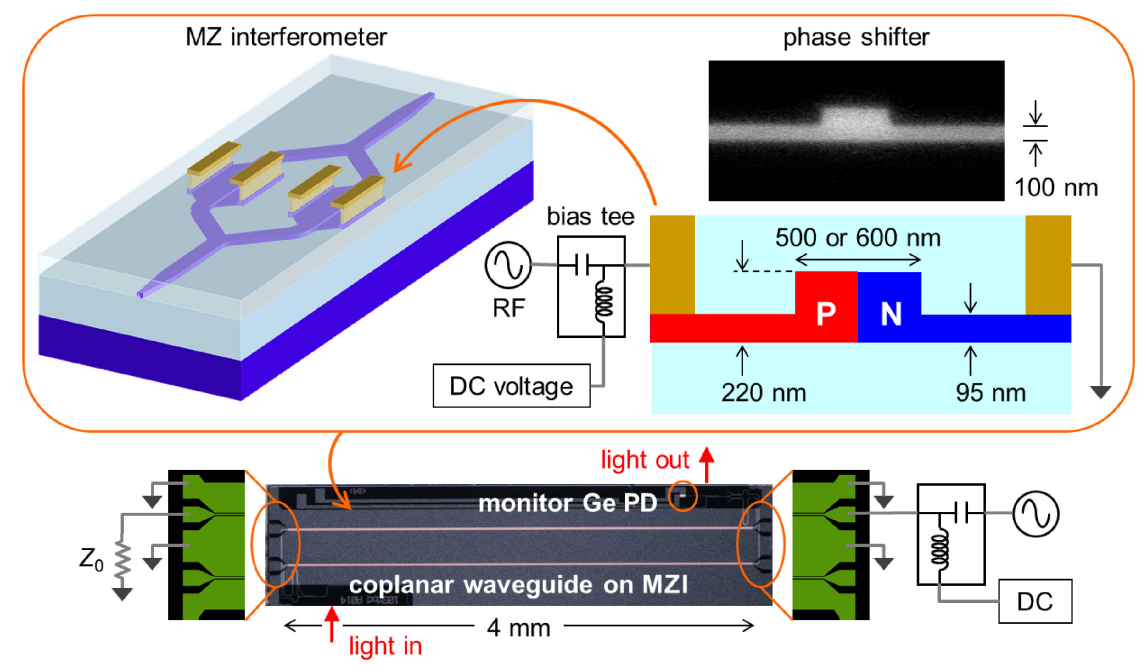

Fig. 1. Silicon $\mathrm{MZ}$ modulator having lateral $\mathrm{PN}$-junction phase shifters. External drive circuits to the modulator are also illustrated.

The slab height has significant influence on high-speed response because of series resistance in $L R C$ coupling described in the next section. Higher slab provides lower series resistance and faster phase modulation. Optical loss is higher due to weaker mode confinement in the center rib area with the slab height higher than $95 \mathrm{~nm}$. A composition image of the rib waveguide in the cross-section photograph was taken by a field-emission scanning electron microscope. It is observed that Si rib waveguide with the precise dimensions was embedded between silica top and bottom clads. The modulators were fabricated on 8-inch SOI wafers of 220-nm SOI thickness and 2- $\mu \mathrm{m}$ buried-oxide (BOX) thickness using 130-nm node CMOS processes. The BOX layer serves as the bottom clad.

Capacitive coupling of reverse-biased PN junction was included in simulation of CPW to obtain a signal electrode of $10-\mu \mathrm{m}$ width and gaps of $6.4-\mu \mathrm{m}$ width between signal and ground electrodes for $50-\Omega$ impedance matching. The RF electrodes were made of $\mathrm{Al}$ with 2- $\mu \mathrm{m}$ thickness and deposited on 2- $\mu \mathrm{m}$ thick top clad. In Fig. 1, DC reverse bias for carrier depletion and RF signal for optical modulation were applied to one arm of the MZI through a signal electrode of the 
CPW with a bias tee. In push-pull operation for zero-chirp modulation, RF signals of opposite polarities with DC reverse bias of the same polarity were applied to the both arms, respectively [13]. The signal electrode was connected to P-doped slab of the rib waveguide, and one of the ground electrodes to N-doped slab of the rib waveguide through $\mathrm{Al}$ vertical interconnect access. The $\mathrm{CPW}$ was designed using short and straight signal electrodes without bending. The signal electrodes were deposited only on top of the phase shifters and connected with contact pads disposed on the both sides. The signal and ground electrodes were wire bonded to external electrodes on package.

To avoid input and output optical fibers interfering with the RF bonding wires, input and output mode-field converters (MFCs) consisting of inverted taper $\mathrm{Si}$ waveguides were located on the side facets using high-index-contrast $\mathrm{Si}$ channel waveguide bends of $25-\mu \mathrm{m}$ bending radius. Optical loss due to the waveguide bends is as low as $0.04 \mathrm{~dB}$ per semicircle [17]. A monolithic monitor Ge PD, which was integrated with a high-index-contrast mode splitter waveguide, allows low-loss performance monitoring of the MZ modulator [18].

\section{Fundamental characteristics}

\subsection{DC characteristics of phase shifter}

DC voltage dependences of phase shift and carrier-induced optical loss are related to $\pi$-shift voltage $V_{\pi}$ and residual carrier-induced intensity modulation, respectively. Phase shift and carrier-induced optical loss were obtained by measuring peak and dip shifts of optical power output from a Si MZ modulator as shown in Fig. 1 with DC reverse-bias voltage applied one arm of MZI. Measured phase shift and carrierinduced optical loss per unit length vs DC reverse-bias voltage are plotted in Fig. 2.
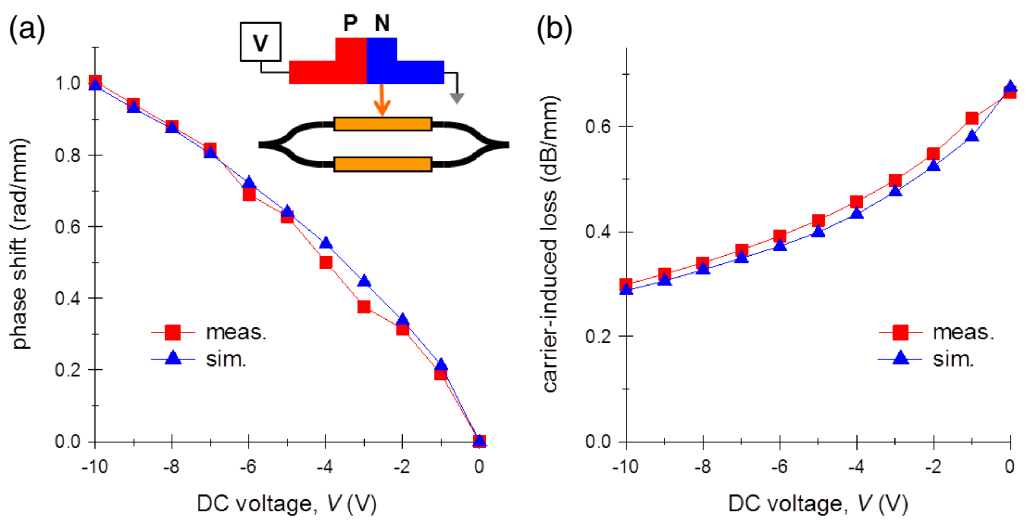

Fig. 2. Experimental and numerical DC voltage dependences of phase shift per unit length (a) and carrier-induced optical loss per unit length (b).

Two-dimensional DC simulation based on a finite-element solver for charge continuity and carrier transport equation combined with Poisson equation was performed to obtain carrier distribution profile in the lateral PN junction under reverse-bias conditions. Dopant profile similar to that presented in the literature was adopted [19]. Electron and hole concentrations in $\mathrm{N}$ and $\mathrm{P}$ regions are 
$2 \times 10^{17} \mathrm{~cm}^{-3}$ and $5 \times 10^{17} \mathrm{~cm}^{-3} ; 1 \times 10^{20} \mathrm{~cm}^{-3}$ in $\mathrm{N}+$ and $\mathrm{P}+$ regions under $\mathrm{Al}$ contacts. Carrier distribution profile was converted to refractive index profile in the rib waveguide using the empirical formula on the free-carrier plasma dispersion $[10,19]$. Higher free-carrier concentration leads to lower refractive index in crystalline Si.

Phase shift and carrier-induced optical loss were derived after light-propagation simulation in the rib-waveguide phase shifter using the simulated refractive index profile by beam propagation method [20]. Simulated phase shift and carrier-induced optical loss as plotted in Fig. 2 coincide well with the experimental results. The phase shifter was fabricated with the precise specifications as designed. Further reduction in $V_{\pi}$ and enhancement of phase shifter efficiency by design refinement on doping profile and numerical analysis as described above are realistic. A voltage-length product defined as $V_{\pi} \ell$ is a measure of phase shifter efficiency, obtained as $2.5 \mathrm{~V} \cdot \mathrm{cm}$ at a reverse bias of $-5 \mathrm{~V}$. Residual intensity modulation due to the carrier-induced optical loss is negligible according to the analysis in the literature [13]. Lateral PN-junction carrier-depletion Si MZ modulators are, therefore, promising as modulators in PSK.

\subsection{Simulated high-speed response of phase shifter}

High-speed response of effective refractive index $n_{\text {eff }}$ was analyzed numerically by time-dependent simulation of the carrier-transport and Poisson equations with input of a linear-ramp electric pulse having 1-ps fall and rise times and 7- $\mathrm{V}_{\mathrm{pp}}$ amplitude at -5 -V DC reverse bias as shown in Fig. 3 [20]. The rise and fall times of 1 ps were deliberately chosen to expose the slower transients of the actual phase shifter, which is $3-6.5$ times slower. Rise and fall times $\tau_{\mathrm{R}}$ and $\tau_{\mathrm{F}}$ in $n_{\text {eff }}$ were obtained as $3.2 \mathrm{ps}$ and $6.6 \mathrm{ps}$ for $10-90 \%$ change in $n_{\text {eff }}$, respectively. Transients of $n_{\text {eff }}$ are governed by dynamics of electrons and holes in the reverse-biased PN junction in the rib-waveguide core.

(a)

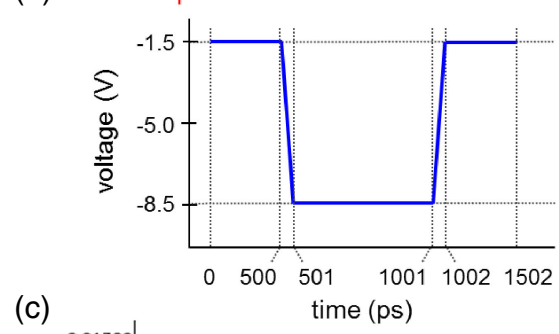

(c)
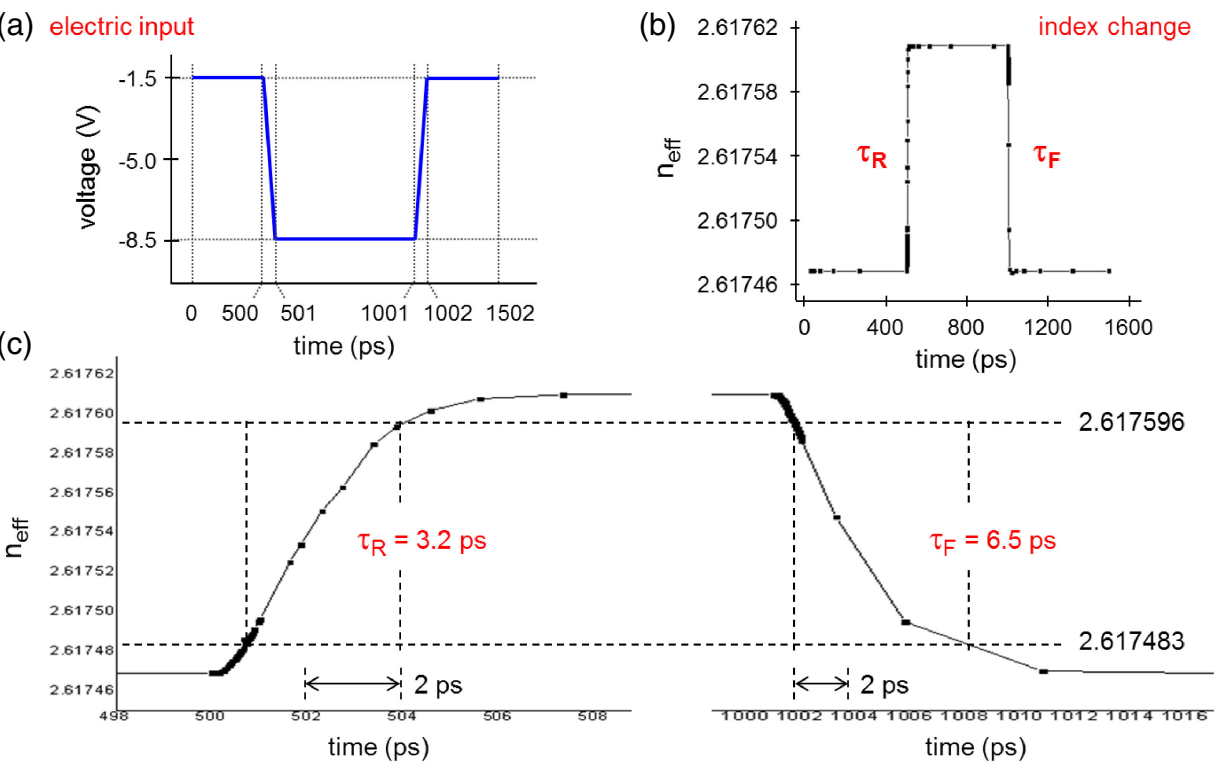

Fig. 3. (a) Linear-ramp electrical input pulse trace. (b) Simulated transient trace of $n_{\text {eff }}$. (c) Rise and fall edges in (b). 
Rise in $n_{\text {eff }}$ is caused by sweeping the charged carriers out of the junction boundary (depletion process), while fall in $n_{\text {eff }}$ is caused by pumping up the charged carriers toward the junction boundary (recovery process). In the recovery process, dense carriers are loaded up into the core. A period of time is elapsed for relaxation of the loaded carriers into equilibrium distribution under the reverse bias via local diffusion. In case of the depletion process, relaxation of the dilute carriers via local diffusion is negligible and does not contribute to refractive-index change. On the other hand, transit times in carrier drift are substantially same in the both processes, which are reverse processes of each other under constant negative and positive electric fields of the same strength, respectively. Therefore, the fall is slower because of the local diffusion than the rise, namely $\tau_{\mathrm{F}}>\tau_{\mathrm{R}}$.

The response times in the present simulation results are much shorter than $10 \mathrm{ps}$, thereby the lateral $\mathrm{PN}$-junction carrier-depletion phase shifter is capable of phase modulation beyond 100-Gbaud symbol rate. Further speed enhancement in lateral PN-junction carrier-depletion rib-waveguide phase shifters will be realized by size shrinkage of the phase shifters in deep-submicron scale to shorten transport time elapsed for carrier drift and for local carrier diffusion. For size shrinkage, improvement in fabrication processes is indispensable to eliminate optical loss caused by side-wall roughness.

Eye diagram in 10-Gb/s NRZ-OOK in Fig. 4 was computed in the timedependent simulation using random bit stream of 500 bits as an input electrical signal stream under optical interference with reference continuous-wave light at a wavelength of $1550 \mathrm{~nm}$ [20]. Amplitude fluctuation of 15-dB signal-to-noise ratio (SNR) was introduced to the input signal stream. High-contrast NRZ-OOK modulation is observed with clear eye opening and extinction ratio $E R>=10 \mathrm{~dB}$.

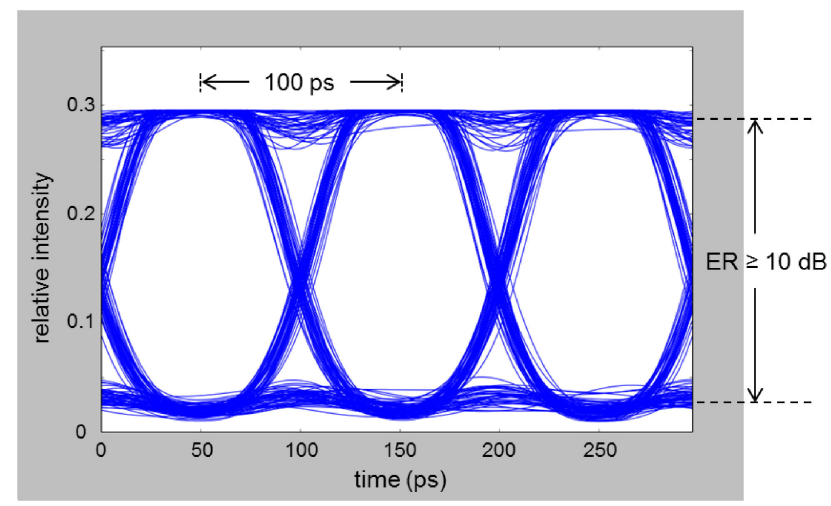

Fig. 4. Simulated eye diagram in $10-\mathrm{Gb} / \mathrm{s}$ NRZ OOK.

After suppression of the local diffusion by the size shrinkage as described above, ultimate limit of the carrier relaxation to the equilibrium distribution will be evident, which is due to plasma oscillation with frequency $v_{\mathrm{P}}$ represented as

$$
v_{\mathrm{P}}^{2}=\frac{4 \pi q^{2}}{\varepsilon}\left(\frac{N}{m_{\mathrm{e}}}+\frac{P}{m_{\mathrm{h}}}\right)
$$


second period is derived for plasma oscillation with $N$ and $P \sim 1 \times 10^{17} \mathrm{~cm}^{-3}$ and the density-of-state effective masses for electrons and holes [22]. Speed limitation by plasma oscillation is considerable only beyond 1-Tbaud symbol rate.

\subsection{Experimental characterization of modulator eye diagrams}

In the traveling-wave $\mathrm{MZ}$ modulator, RF frequency response of the traveling-wave electrodes and velocity mismatch between the guided light and the RF signal wave have significant influences on optical-modulation speed. Extensive study has been devoted to traveling-wave lateral PN-junction carrier-depletion Si MZ modulators in major modulation formats in OOK and PSK [6, 7, 12, 13, 16, 23, 24, 25, 26, 27]. Some of the results reported for high-speed performances in NRZ OOK are listed in Table I.

Table I. Performances of traveling-wave lateral PN-junction Si MZ modulators.

\begin{tabular}{c|c|c|c|c|c}
\hline bit rate & $E R$ & $V_{\pi} \ell$ & $\ell$ & on-chip loss & reference \\
\hline $40 \mathrm{~Gb} / \mathrm{s}$ & $10 \mathrm{~dB}$ & $2.7 \mathrm{~V} \cdot \mathrm{cm}$ & $3.5 \mathrm{~mm}$ & $15 \mathrm{~dB}$ & {$[23]$} \\
\hline $30 \mathrm{~Gb} / \mathrm{s}$ & $8.5 \mathrm{~dB}$ & $1.86 \mathrm{~V} \cdot \mathrm{cm}$ & $6 \mathrm{~mm}$ & $9 \mathrm{~dB}$ & {$[24]$} \\
\hline $32 \mathrm{~Gb} / \mathrm{s}$ & $11.3 \mathrm{~dB}$ & $2.5 \mathrm{~V} \cdot \mathrm{cm}$ & $4 \mathrm{~mm}$ & $5.5 \mathrm{~dB}^{*}$ & {$[25]$} \\
\hline
\end{tabular}

*Derived from 10.5-dB total insertion loss and 2.5-dB MFC coupling loss per facet.

Eye-diagram measurements in NRZ OOK were performed for the Si single MZ modulator, which consists of the Si MZI having 4-mm phase shifters as shown in Fig. 1, at bit rates of $10-32 \mathrm{~Gb} / \mathrm{s}$ [25]. Bit rate is equal to symbol rate in OOK. Measured extinction ratio, as plotted in Fig. 5, decreases with increasing bit rate; $13.9 \mathrm{~dB}$ at $10 \mathrm{~Gb} / \mathrm{s}$ and $11.3 \mathrm{~dB}$ at $32 \mathrm{~Gb} / \mathrm{s}$. The bit-rate dependence of $E R$ is limited by RF loss of the CPW as elaborated in the next subsection.

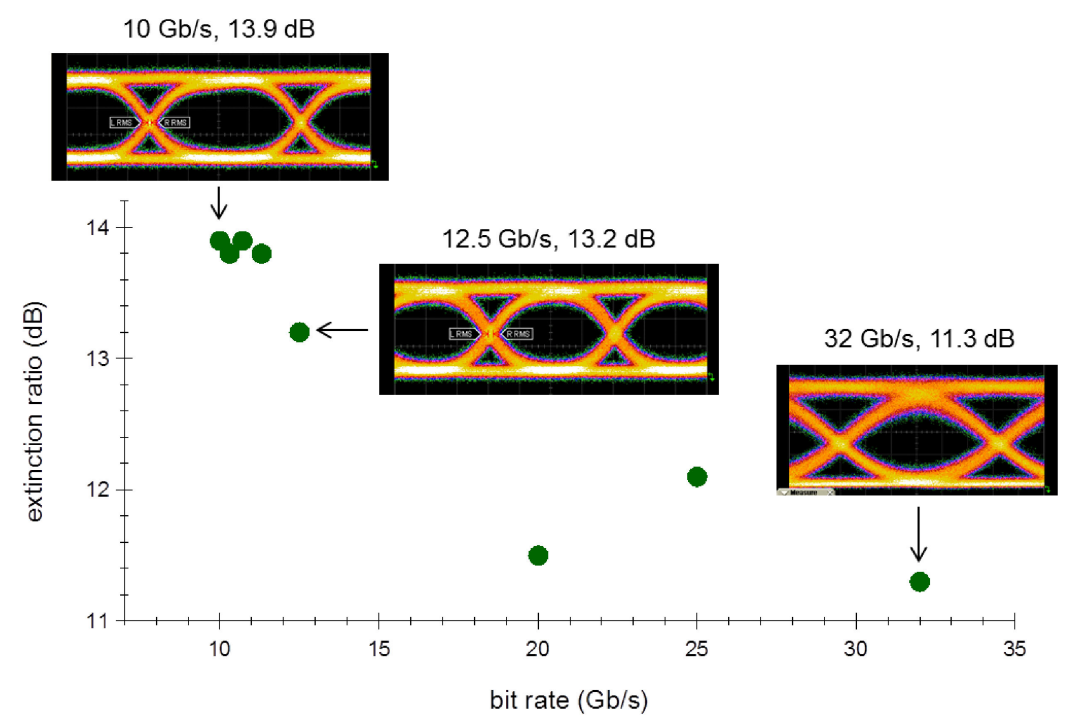

Fig. 5. Extinction ratio vs bit rate obtained for experimental eye diagrams in NRZ OOK at $10-32 \mathrm{~Gb} / \mathrm{s}$. Some eye diagrams are also presented. 


\subsection{Frequency responses of phase shifter with traveling-wave electrodes}

$\mathrm{RF}$ loss per unit length and impedance were obtained in electrical S-parameter measurements of 4-mm traveling-wave Si rib-waveguide phase shifter [25]. RFloss and impedance data acquired at DC reverse-bias voltages of $0 \mathrm{~V}$ and $-5 \mathrm{~V}$ are shown in Fig. 6. The loss peak is caused by series $L R C$ resonance: inductance $L$ from $\mathrm{Al}$ interconnects, resistance $R$ from Si side slabs and capacitance $C$ from PN junction. The $L R C$ resonance frequency shifts from $22.0 \mathrm{GHz}$ at $0 \mathrm{~V}$ to $35.7 \mathrm{GHz}$ at $-5 \mathrm{~V}$, because the junction capacitance is decreased with increasing depletion width under higher reverse bias [6]. As a result of the resonance frequency shift, RF loss in a frequency range lower than $26 \mathrm{GHz}$ is reduced at a DC voltage of $-5 \mathrm{~V}$. Equivalent circuit of the traveling-wave phase shifter including $L R C$ elements in series is presented in Fig. 6. In conventional equivalent circuit models for lateral PN-junction carrier-depletion Si phase shifters, $R C$ coupling only was taken into account [28].

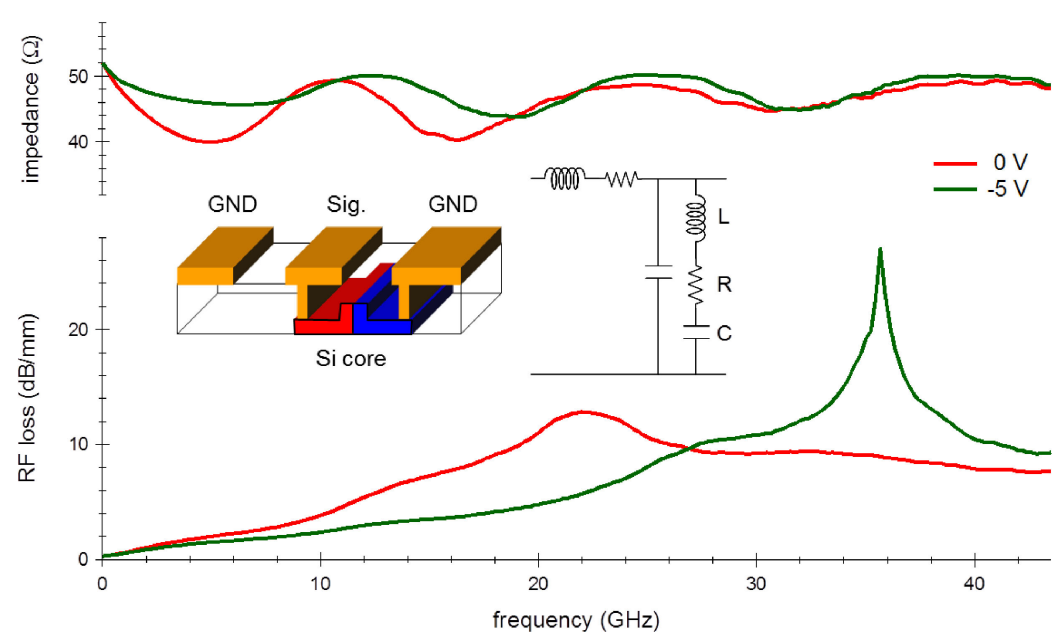

Fig. 6. Measured RF loss per unit length (bottom graph) and impedance (top graph). Inset: perspective illustration of traveling-wave lateral $\mathrm{PN}$-junction $\mathrm{Si}$ phase shifter and its equivalent circuit.

Electro-optic (EO) frequency responses of Si single MZ modulators having 3$\mathrm{mm}$ and 5-mm phase shifters were obtained in EO S-parameter measurements at various DC reverse-bias voltages as shown in Fig. 7, where normalized optical response is plotted as a function of RF frequency. For applications to modularized Si MZ modulators presented in the next section, low-loss Si rib-waveguide phase shifters with compensated side-wall regions, as illustrated in Fig. 7, were introduced into Si MZI waveguides. Carrier concentration in the side wall regions was decreased by compensation doping to reduce carrier-induced optical loss [29]. Carrier doping profile around the junction boundary was unchanged and the compensated lateral $\mathrm{PN}$-junction phase shifter operates as the uncompensated lateral PN-junction phase shifter. High-frequency responses were not deteriorated by compensation doping, while carrier-induced optical loss was decreased by $40 \%$. 


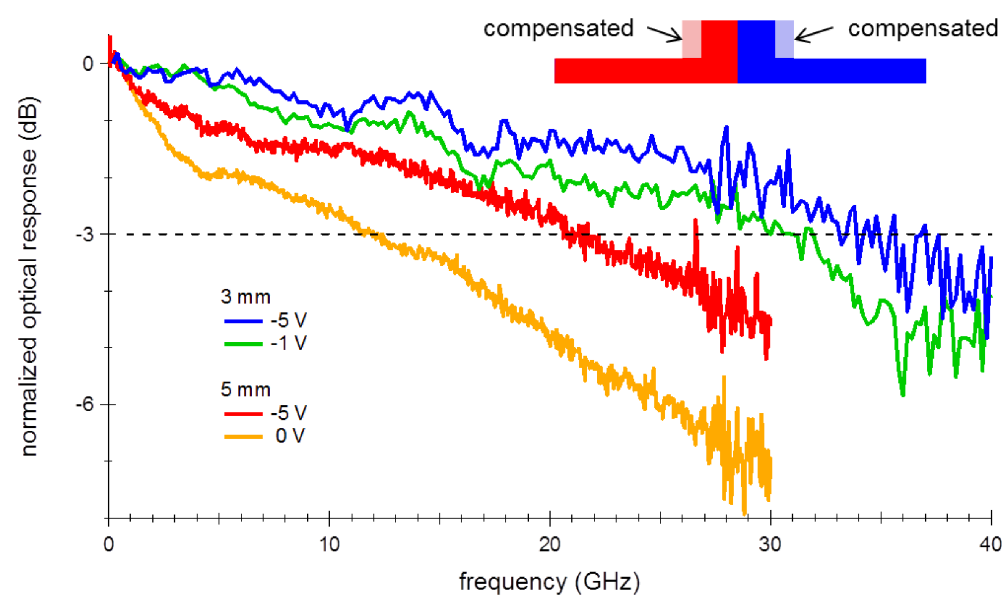

Fig. 7. Measured EO response of single $\mathrm{MZ}$ modulator with 3-mm phase shifter at DC voltages of $0 \mathrm{~V}$ and $-5 \mathrm{~V}$ and with $5-\mathrm{mm}$ phase shifter at DC voltages of $0 \mathrm{~V}$ and $-5 \mathrm{~V}$. Inset: illustrated profile of PN junction in rib-waveguide phase shifter with compensated side walls.

Frequency of 3-dB roll-off for 3-mm phase shifter is $30.6 \mathrm{GHz}$ at $-1 \mathrm{~V}$ and $33.4 \mathrm{GHz}$ at $-5 \mathrm{~V}$, while $12.2 \mathrm{GHz}$ at $0 \mathrm{~V}$ and $21.3 \mathrm{GHz}$ at $-5 \mathrm{~V}$ for $5-\mathrm{mm}$ phase shifter. The EO responses are dominated by the RF loss shown in Fig. 6. Higher 3$\mathrm{dB}$ roll-off frequency is obtained with shorter phase shifter length because of lower RF loss; higher 3-dB roll-off frequency also obtained at higher reverse bias because of smaller junction capacitance and hence higher $L R C$ resonance frequency. On the other hand, longer phase-shifter length is required for lower $V_{\pi}: 7-8 \mathrm{~V}$ with 3-mm phase shifter and 6-7 V with 5-mm phase shifter.

\section{Applications to high-speed optical-fiber transmission}

\subsection{0-Gb/s NRZ OOK}

For application to transmission in 10-Gb/s NRZ OOK, modularized Si modulators were fabricated using Si MZ modulator (MZM) chips having single MZIs described in Section 2 and Fig. 1. Silicon MZM chips with 5-mm compensation-doped phase shifters were used for this application, because 3-dB EO roll-off frequency of $21 \mathrm{GHz}$ at $-5-\mathrm{V}$ DC reverse bias is sufficient to cover the frequency bandwidth of $10-\mathrm{Gb} / \mathrm{s}$ NRZ OOK. Hermetically sealed ceramic-based metal packages in dimensions of $28 \mathrm{~mm}(\mathrm{~L}) \times 10 \mathrm{~mm}(\mathrm{~W}) \times 8 \mathrm{~mm}(\mathrm{H})$ were prepared to accommodate $\mathrm{Si}$ MZM chips [17].

Ceramic-based packaging has advantages of high-reliability packaging and high thermal stability in modulator performance because of thermal expansion coefficient of ceramics close to that of crystalline silicon. Each Si MZM chip was mounted on a chip carrier as depicted in Fig. 8. Metal contact pads on an edge of a Si MZM chip were connected to coplanar electrodes on a chip carrier by wire bonding. The coplanar electrodes on the chip carrier were interconnected to feedthrough electrodes. Contact pads on the other-side edge of the Si MZM chip were wire bonded to a $50-\Omega$ terminator and electric grounds on the chip carrier. Aspheric lenses were mounted for low-reflection coupling to input and output 


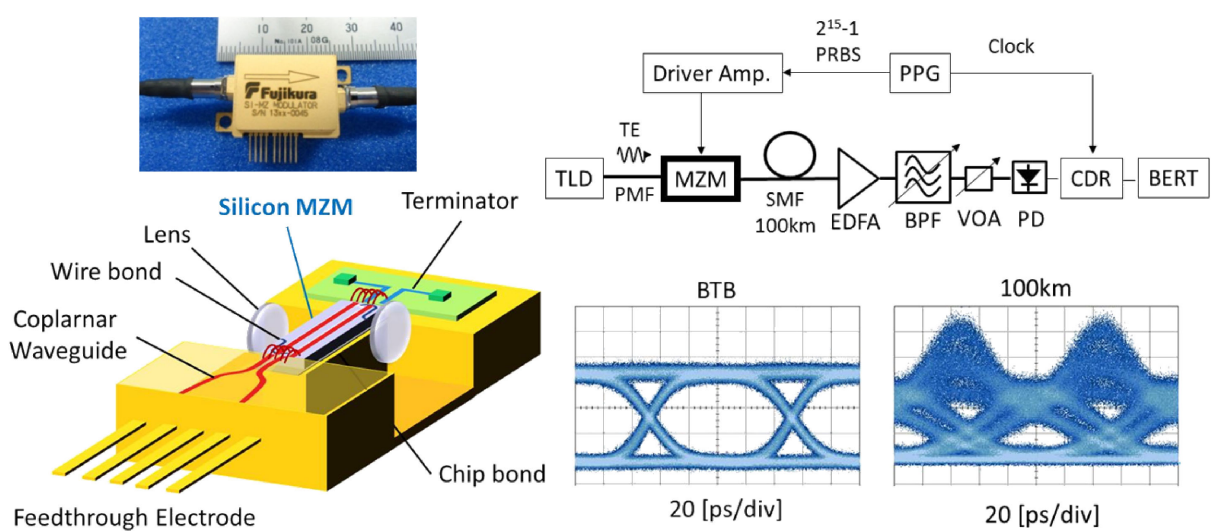

Fig. 8. Silicon $M Z$ optical modulator (MZM) in package and its eye diagrams in BTB and 100-km SMF transmission in $10-\mathrm{Gb} / \mathrm{s}$ NRZ OOK. Block diagram of set-up for 100-km SMF transmission measurement is shown.

optical fibers. Angled Si inverted taper MFCs were integrated with Si MZI to avoid reflection back to the fibers [17]. Return loss as high as $40 \mathrm{~dB}$ was achieved without anti-reflection coating on chip facets. Optical insertion loss was obtained lower than $14 \mathrm{~dB}$ for the fabricated Si MZM packages. Further reduction in optical insertion loss below $11 \mathrm{~dB}$ is possible with refinement in Si MFC design and lens mounting technology.

Transmission in 100-km standard-dispersion single-mode fiber (SMF) link was performed using the set-up in Fig. 8. Continuous-wave light of 1550-nm wavelength from a tunable laser diode (TLD) in TE polarization was input to a modularized Si MZM through a polarization maintaining fiber (PMF). Electrical signals in $2^{15}-1$ pseudo-random bit stream (PRBS) from a pulse pattern generator (PPG) and a driver amplifier were applied to a phase shifter in one arm of MZI. For bit-error-rate (BER) measurement as a function of optical SNR, amplified spontaneous emission from an Er-doped fiber amplifier (EDFA) was filtered with a bandpass filter (BPF) in a range of $+/-0.5 \mathrm{~nm}$ around the laser wavelength and attenuated with a variable optical attenuator (VOA) [30]. Optical modulation signals were detected with a high-speed PD. Signal clock was extracted by using a clock data recovery (CDR) unit with reference clock from PPG and a BER tester (BERT) was used for BER measurements.

In BER measurements, power penalty at $10^{-3}$ BER in 100-km SMF transmission was obtained as low as $1.2 \mathrm{~dB}$ with respect to BER in back to back (BTB). Eye diagrams in BTB and 100-km SMF transmission as shown in Fig. 8 were acquired by a sampling oscilloscope instead of BERT with 14-dB ER for BTB eyediagram.

\section{$4.2128-G b / s$ DP-QPSK}

Silicon MZMs incorporating 3-mm phase shifters for IQ modulation at 32-Gbaud were fabricated for application to 128-Gb/s DP-QPSK as shown in Fig. 9. The Si MZM consists of two Si in-phase quadrature (IQ) MZMs and a waveguide circuit for polarization-division multiplexing (PDM). 


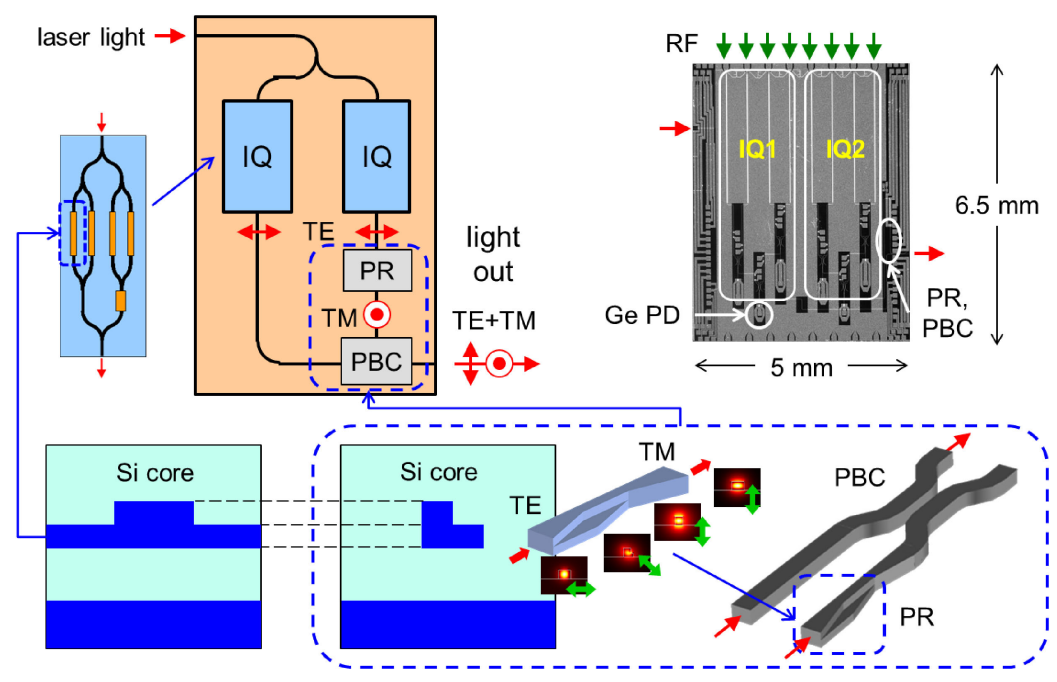

Fig. 9. Schematic illustration and top-view photograph of monolithic Si PDM IQ MZM.

A Si PDM IQ MZM consists of the following four essential blocks: (1) an input waveguide split into two parallel waveguides with a multi-mode interferometer, (2) two Si IQ MZM, each of which consists of a Si nested MZI incorporating Si single sub-MZIs on both of parent MZI arms and operates in TE polarization state, (3) a polarization rotator (PR), with which polarization of light after one of the IQ MZMs is converted to transverse-magnetic (TM) polarization and (4) a polarization beam combiner (PBC) to multiplex TE and TM modulator outputs [27]. A Si PDM IQ MZM was monolithically integrated in a footprint as small as $6.5 \times 5 \mathrm{~mm}^{2}$ with Ge PDs for performance monitoring and $\mathrm{Si}$ rib-waveguide thermo-optic phase controllers to adjust phase difference to $\pi$ between I and Q components in each polarization. Rib-waveguide-based PR in PDM circuit was designed with common design rule with the rib-waveguide phase shifter, thereby suitable for high-yield low-cost fabrication.

A Si PDM IQ MZM was mounted in a ceramic-based metal package with four modulator drivers in dimensions of $35 \times 15 \times 4.5 \mathrm{~mm}^{3}$ as shown in Fig. 9. Butt fiber coupling with $2.5-\mathrm{dB}$ coupling loss, $1-\mathrm{dB}$ polarization-dependent loss and 40$\mathrm{dB}$ return loss was achieved for low-profile packaging by using suspended MFCs [31]. Optical insertion loss was measured as lower than $15 \mathrm{~dB}$ in $\mathrm{C}$ band.

Long-haul transmission measurements were performed using 100-km SMF loop link as illustrated in Fig. 10 [32]. Two optical switches (SWs) allowed synchronized burst-mode loop transmission in 50 and 100 loop turns to reach $500-\mathrm{km}$ and $1000-\mathrm{km}$ total spans, respectively. Transmitted optical signals were detected with a coherent receiver and demodulated with an offline digital signal processor (DSP) to obtain constellation diagrams in two orthogonal linear polarization states [4]. 

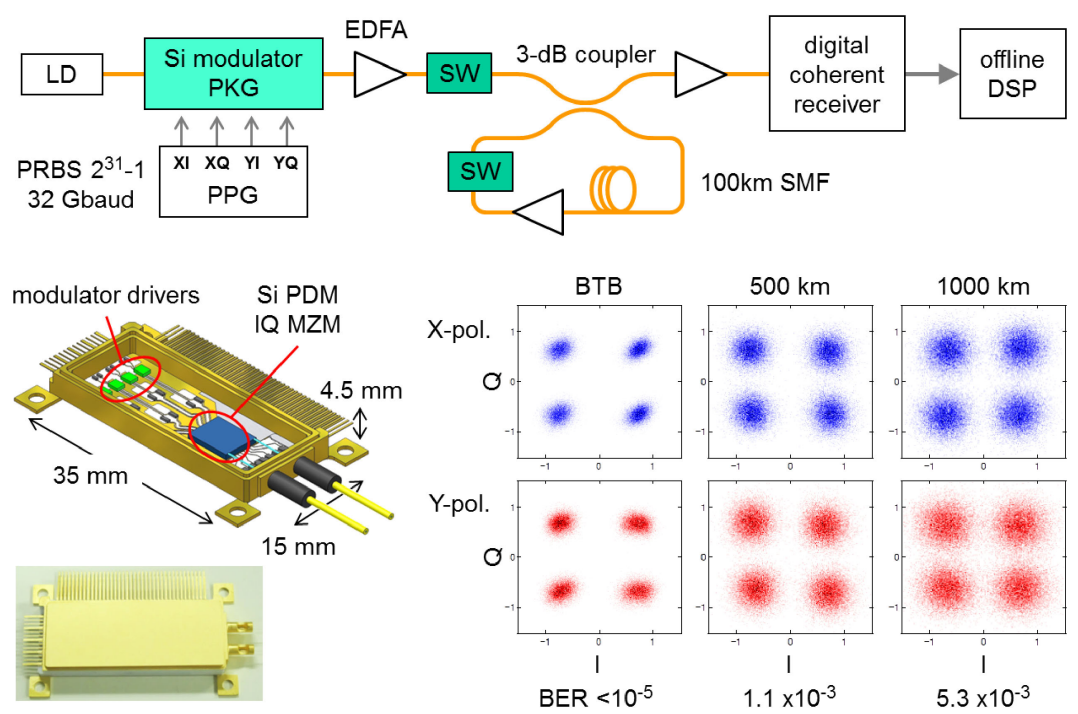

Fig. 10. Modularized Si MZ modulator in package with modulator drivers inside for $128-\mathrm{Gb} / \mathrm{s}$ DP-QPSK, its constellation diagrams in X- and Y-polarization states up to $1000-\mathrm{km}$ fiber transmission, block diagram for transmission set-up including 100-km SMF loop link.

\section{Conclusion}

Fundamental characteristics of the lateral PN-junction carrier-depletion Si MZ modulators were reviewed on the basis of experimental and numerical analyses. Optical-fiber transmission in 10-Gb/s NRZ OOK and 128-Gb/s DP-QPSK was demonstrated using the Si MZ modulators modularized in ceramic-based metal packages.

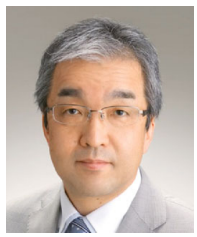

\section{Kensuke Ogawa}

received the B.S. degree in physics from Osaka City University in 1982, the M.S. and Ph.D degrees in physics in 1984 and 1987 from Osaka University. In 1987, he joined Central Research Laboratory, Hitachi Ltd., where he performed research on semiconductor optoelectronics devices. During 1991 to 1993, he stayed in Hitachi Cambridge Laboratory, UK for research on semiconductor quantum devices. Since 2006, he has been with Optics and Electronics Laboratory, Fujikura Ltd. His current research subjects include silicon photonics and optical performance monitoring. He is a member of IEICE and JSAP, and a senior member of OSA.

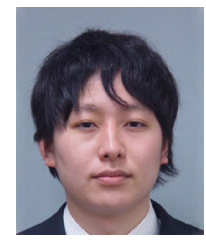

\section{Hiroki Ishihara}

received the B.S and M.S degrees in communications engineering from Tohoku University, Sendai, Japan, in 2010 and 2012, respectively. Since 2012, he has been with Optics and Electronics Laboratory, Fujikura Ltd, where he has been engaged in research and development of silicon photonic devices for optical communication. 


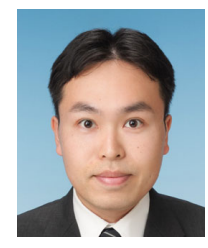

\section{Kazuhiro Goi}

received the B.S. and M.S. degrees in physics from Waseda University, Tokyo, Japan, in 2002 and 2004, respectively. During 2004 to 2007, he was with Matsushita Electric Industrial Co. Ltd. From 2007 to present, he now with Fujikura Ltd, where he has been engaged in research and development of silicon photonic devices for optical communication.

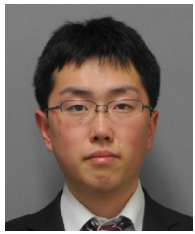

\section{Yasuhiro Mashiko}

received the B.E. and M.E. degrees in electrical and electronic engineering from Tokyo Institute of Technology, Tokyo, Japan, in 2010 and 2013, respectively. In 2013, he joined the Fujikura Ltd., Koto-ku, Tokyo, Japan, where he has been engaged in research and development of silicon photonic devices for optical communication.

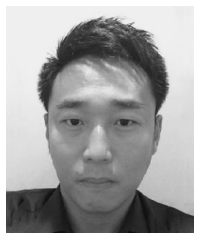

\section{Soon Thor Lim}

received his Ph.D. from the University of Surrey under the Agilent Technologies, Singapore Scholarship in 2005 and First Class Honors in Electronic and Electrical Engineering in 2001. He has more than 12 years experiences in photonics related research and is currently with the Institute of High Performance Computing A*STAR in Singapore. He is also seconded to Optic2Connect Pte Ltd as technical manager.

He has won numerous awards, including, the IET Innovation Award 2011 - Software and Design Category (Highly Commended) in the United Kingdom for his work in modeling and simulation of high speed optical devices. He holds several patents and published numerous scientific journal and conferences.

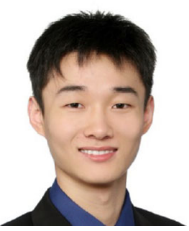

\section{Min Jie Sun}

received the B.E. degree in Electrical Engineering from National University of Singapore, in 2013. In 2014, he joined Optic2Connect Pte Ltd, Singapore, where he has been engaged in the research and development of simulation and modelling techniques for silicon optical modulators.

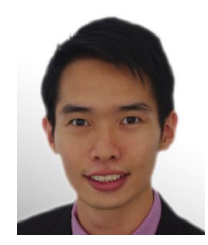

\section{Sean Seah}

received the B.E. degree in Electrical Engineering from National University of Singapore, in 2007. In 2014, he joined Optic2Connect Pte Ltd, Singapore, providing technical solutions for silicon photonics. His past experience was in avionics engineering and technology commercialisation.

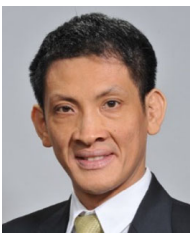

\section{Ching Eng (Jason) Png}

received his Ph.D. degree in Silicon Photonics from Surrey University in 2004, executive MBA degrees from INSEAD and Tsinghua University in 2014. He also completed the Innovative Business Leadership Program at Massachusetts Institute of Technology (MIT) Sloan School in 2013. He was with Agilent Technologies from 1999 to 2000. Since 2005, he is with Institute of High Performance Computing (IHPC) A*STAR in Singapore.

He co-founded Optic2Connect Pte Ltd to commercialize optical simulations developed at IHPC and are funded by Skolkovo Foundation and Spring Singapore. 

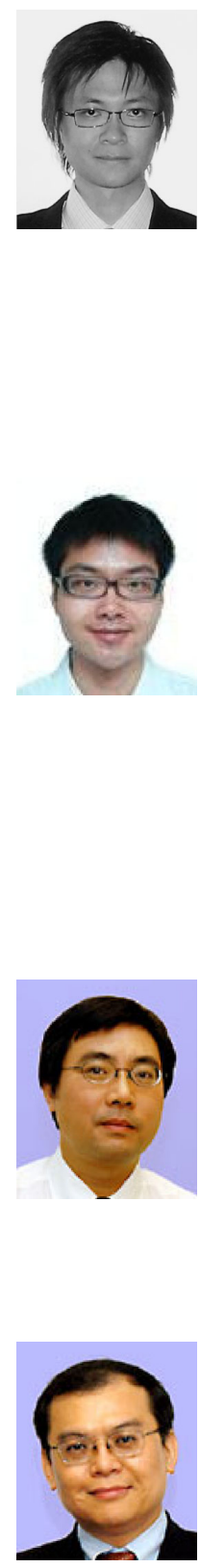

\section{Dim-Lee Kwong}

is the Executive Director of Institute of Microelectronics (IME), Agency for Science, Technology and Research (A*STAR), Singapore and a Professor of Electrical and Computer Engineering at National University of Singapore. He was Earl N. and Margaret Bransfield Endowed Professor of Electrical and Computer Engineering at The University of Texas at Austin from 1990-2007 and the Temasek Professor of National University of Singapore from 2001-2004.

Prof. Kwong received IBM Faculty Award in 1984-86, Semiconductor Research Corporation (SRC) Inventor Awards in 1993-94, General Motor Foundation Fellowship in 1992-95, Halliburton Foundation Excellent Teaching Award in 1994, Engineering Foundation Award in 1995, IEEE George Smith Award in 2007, and the 2011 IEEE Frederik Philips Award with citation: "For leadership in silicon technology and excellence in the management of microelectronics $R \& D$." Prof. Kwong received the Singapore President's Science and Technology Medal, the highest award bestowed on individuals who have made exceptional contributions to the development of Singapore. He was recognised for his distinguished, sustained and exceptional contributions to Singapore's science and engineering landscape, particularly in advancing the semiconductor industry through $\mathrm{R} \& \mathrm{D}$, forging of strategic research partnerships, and enhancing competitiveness of Singapore's semiconductor ecosystem towards a knowledge-based economy. Prof. Kwong is an IEEE Fellow. 